\title{
Antigen-targeted Personalized Breast Cancer Vaccine
}

National Cancer Institute

\section{Source}

National Cancer Institute. Antigen-targeted Personalized Breast Cancer Vaccine. NCI

Thesaurus. Code C120039.

An individualized, therapeutic cancer vaccine (IVAC) composed of liposomes containing RNA encoding two or three tumor associated antigens (TAAs) that are specifically expressed in the patient's individual cancer selected from a warehouse ("off the shelf") and p53 RNA, with potential immunostimulatory and antineoplastic activities. Upon administration, the antigen-targ eted personalized breast cancer vaccines are translated by antigen presenting cells (APCs) and the expressed protein is presented via major histocompatibility complex (MHC) molecules on the surface of the APCs. This leads to an induction of both cytotoxic T-lymphocyte ( $C T L$ ) and memory T-cell immune responses against the TAAs. The RNAs in the vaccine are specifically selected for an individual patient after RNA profiling of the patient's tumor. 